\title{
Studies on Genetic Parameters, Correlation and Path Analysis for Yield and Yield Attributing Traits in Sweet Corn (Zea mays L. saccharata)
}

\author{
Sonal Chavan $^{1 *}$, D. Bhadru ${ }^{2}$, V. Swarnalatha ${ }^{3}$ and B. Mallaiah ${ }^{2}$ \\ ${ }^{1}$ Department of Genetics and Plant breeding, College of Agriculture, Rajendranagar, \\ Professor Jayashankar Telangana State Agricultural University, Hyderabad-500030 \\ ${ }^{2}$ Maize Research Centre, Rajendranagar, Hyderabad, India \\ Seed Research and Technology Centre, Rajendranagar, Hyderabad, India
}

*Corresponding author

\begin{tabular}{l} 
K e y w o r d s \\
$\begin{array}{l}\text { Sweet corn, Genetic } \\
\text { parameters, } \\
\text { Correlation, Path } \\
\text { analysis, Sugar } \\
\text { content and PFSR }\end{array}$ \\
\hline Article Info \\
$\begin{array}{l}\text { Accepted: } \\
\text { 14 June } 2020 \\
\text { Available Online: } \\
\text { 10 July } 2020\end{array}$ \\
\hline
\end{tabular}

A B S T R A C T

The present investigation was carried out in 25 sweet corn inbred lines during Rabi 2019-20. Significant differences among genotypes for most of the traits were noticed, indicating the presence of substantial genetic variability. Phenotypic coefficients of variation (PCV) were higher than genotypic coefficients of variation $(\mathrm{GCV})$, indicating the role of experimental variance to the total variance. Moderate to high range of GCV, PCV, heritability and genetic advance over mean were recorded for ear height, ear length, number of rows per ear, number of kernels per row, cob weight with husk and without husk and PFSR disease score, which indicates the importance of these traits in selection of sweet corn inbred lines. Ear height, ear length, number of rows per ear, number of kernels per row, cob weight without husk were very important for selection of sweet corn genotypes as they recorded positive significant correlations and direct effects were also recorded by some of these traits on cob weight with husk i.e. green cob yield.

\section{Introduction}

Among the various specialty corns, sweet corn is gaining popularity in India and other Asian countries. It also has a very huge market potential not only in national market but in global market as well. Fresh and raw sweet corn ears are consumed after cooking as well as in roasted form and are increasingly in high demand in the hotels for the preparations of delicious sweet corn soup. Sweet corns are eaten green as highly valued fresh products like baby corn; immature kernels are parboiled and/or dried to produce candy, mature kernels are crushed to produce the confection pinnole as a fermentable source for 
the production of an alcoholic beverage, chichi and also used as material for deriving large number of industrial products such as starch syrup, dextrose and dextrin etc. In India, composites like Madhuri and Priya (ANGRAU), HSC-1, win orange and Punjab Sweet Corn 1 from the public sector and only few hybrids (Sugar 75 and Misti) have been developed by the private sector. No sweet corn hybrid has been released so far by the public sector. The available public cultivars are low yielding, so the farmers have to depend on private sector hybrids which are sold to farmers at high cost. So development of high yielding sweet corn hybrids is need of the hour.

For the development of productive and adapted cultivars to supply the market demand, understanding of genetic variability present in a given crop species for the traits under improvement is imperative for the success of any plant breeding program (Sankar et al., 2006). Genotypic and phenotypic coefficients of variation (GCV and $\mathrm{PCV}$ ) are useful in detecting the amount of variability present in a given characteristic. The efficiency with which genotypic variability can be exploited by selection depends upon heritability and genetic advance (GA) of individual trait (Bilgin et al., 2010).

Heritability is a measure of the phenotypic variance attributable to genetic causes and it predicts the extent to which a particular character can be transmitted to successive generations. Whereas genetic advance shows the degree of gain obtained for the characters under a particular selection pressure (Niji et al., 2018). Heritability estimates along with genetic advance are normally more helpful in predicting gain under selection than heritability estimates alone as it is not sufficiently informative about the existence of gene action (additive/non-additive) and involvement of other factors in the expression of traits (Johnson et al., 1955).
As yield is a dependent character and it is based on number of quantitative characters, it is important to study the association between pairs of these attributes for faster assessment of high yielding genotypes in selection programme. Correlation studies reflect the extent of association between two characters and helps in determining the yield contributing characters. Genotypic correlations reveal the real association between traits, as it does not include the environmental effects as such in case of phenotypic correlations and thereby is more important compared to phenotypic correlation. Correlation coefficients generally show relationships among independent variables and the degree of linear relation between these characteristics but it does not sufficiently predict the success of selection. However, path-coefficient analysis that is originally developed by Wright (1929) is the most valuable tool to establish the exact correlation in terms of cause and effect. It allows one to identify the direct, indirect and total (direct + indirect) causal effect, as well as to remove any spurious effect that may be present (Hefny, 2011).

Keeping in view the significance of these parameters, present investigation was undertaken in sweet corn on genetic parameters, correlation and path coefficient analysis.

\section{Materials and Methods}

The present investigation was carried out in Rabi, 2019-20 at Maize Research Centre, Rajendranagar, Hyderabad, which is located at an altitude of $542.6 \mathrm{~m}$ and at $79^{\circ} 23^{\prime} \mathrm{E}$ longitude and $17^{\circ} 19^{\prime} \mathrm{N}$ latitude. Twenty five sweet corn inbred lines were evaluated in randomized block design with two replications with spacing of $60 \times 10 \mathrm{~cm}$. The observations were collected on thirteen yield and yield contributing traits viz., days to $50 \%$ 
tasseling, days to $50 \%$ silking, plant height $(\mathrm{cm})$, ear height $(\mathrm{cm})$, ear length $(\mathrm{cm})$, ear diameter $(\mathrm{cm})$, number of kernel rows per ear, number of kernels per row, cob weight with husk (kg/ha), cob weight without husk $(\mathrm{kg} / \mathrm{ha})$, total soluble sugars $(\%)$ by using brix meter, green fodder yield ( $\mathrm{kg} / \mathrm{ha})$ and post flowering stock rot (PFSR) disease score (Macrophomina phaseolina) by tooth pick method.

\section{Statistical analysis}

The data collected was subjected to statistical analysis using INDOSTAT software version 9.2 and the methods adopted by the software for the analysis of variance (ANOVA) was as described by Panse and Sukhatme (1985), mean, standard error and range were calculated as per Singh and Chaudhary (1985). Phenotypic and genotypic coefficients of variation (PCV and GCV) were as per Burton (1952) and were categorized as low $(0-10 \%)$, moderate (10-20\%) and high $(>30 \%)$ as indicated by Sivasubramanian and Madhavamenon (1973). Heritability in broad sense was estimated as the ratio of genotypic variance to the phenotypic variance as suggested by Hanson et al., (1956) and it was as categorized as low (0-30\%), moderate (30$60 \%)$ and high $(>60 \%)$ as indicated by Johnson et al., (1955).

Genetic advance (GA) and genetic advance as per cent of the mean (GAM) were calculated by using the formulae given by Johnson et al., (1955). The GA as percent of the mean was categorized as low (0-10\%), moderate (10$20 \%$ ) and high (>20\%) according to Johnson et al., (1955). Correlation coefficients were calculated by using the formulae given by Johnson et al., (1955). The direct and indirect effects for genotypes were estimated by using path coefficient analysis suggested by Wright (1921) and Dewey and Lu (1959).

\section{Results and Discussion}

The analysis of variance revealed significant difference among genotypes for all traits except for the traits like days to $50 \%$ tasseling, plant height and green fodder yield (Table 1) indicating the presence of considerable significant variation among the genotypes selected, which is pre-requisite for the breeder to take up any breeding programme.

In the present study, estimates of PCV were found to be slightly higher than their corresponding GCV for most of the traits (Table 2), which indicates that the expressions of these traits were influenced by environment to a limited extent and there is possibility of improvement of these traits by using phenotypic selection. Similar findings of higher PCV than GCV were reported by Alan et al., (2013), Niji et al., (2018) and Ayodeji Abe and Adelegan (2019) in sweet corn. The difference between PCV and GCV estimates were found to be more for the traits days to $50 \%$ tasseling, plant height and green fodder yield, indicating that for these traits the phenotypic selection may be misleading. Bello et al., (2012), Nzuve et al., (2014) and Sesay et al., (2016) have also reported high difference between PCV and GCV estimates for plant height. Low to medium PCV and GCV estimates were recorded for most of the traits. The trait PFSR disease score, cob weight without husk, cob weight with husk and ear height registered more than 20 percent of GCV and PCV and these traits were considered for selection of sweet corn inbred lines. Hefny (2011) for yield per plant, Reddy et al., (2012) and Meena et al., (2016) for ear height and Niji et al., (2018) for green cob yield reported high estimates of PCV and GCV.

The genetic components of variation together with heritability estimates would give the best 
picture of amount of genetic advance to be expected from the selection (Burton, 1952). Traits days to $50 \%$ silking, ear height, ear length, ear diameter, no. of kernel rows per ear, ear length, no. of kernels per row, cob weight with husk, total soluble sugars and PFSR disease score registered high estimates of broad sense heritability. However, selection for improvement of such characters may not be useful, because broad sense heritability is based on total genetic variance which includes additive, dominant and epistatic variances. Thus, heritability estimates coupled with high genetic advance would be more reliable and useful on correlating selection criteria (Reddy et al., 2012). The traits ear height, ear length, no. of kernel rows per ear, no. of kernels per row, cob weight with husk, cob weight without husk and PFSR disease score exhibited high estimates of genetic advance as per cent of mean.

High heritability accompanied with high genetic advance as per cent of mean were recorded for ear height, ear length, no. of kernel rows per ear, no. of kernels per row, cob weight with husk and PFSR disease score, indicating that the heritability is due to additive gene effects. Moderate heritability with high genetic advance as per cent of mean was recorded for cob weight without husk, indicating the role of additive gene effects, the moderate heritability may be due to environmental effects and selection would be rewarding for this trait. Thus the traits are fixable and selection would be effective for these traits. Similar findings were reported by Reddy et al., (2012) for ear height, ear length and no. of kernels per row, Begum et al., (2016) for ear height, Sesay et al., (2016) for yield and Niji et al., (2018) for green cob weight.

While selecting the sweet corn genotype, priority should be given for characters like ear height, ear length, number of kernels rows per ear, number of kernels per row, cob weight with husk and without husk and PFSR disease score as showed moderate to high range of GCV, PCV, heritability and genetic advance over mean.

Plant height, ear height, ear length, ear diameter, number of kernels per row and cob weight without husk were observed to exhibit significant positive correlations with cob weight with husk at phenotypic and genotypic level (Table 3). Whereas, number of rows per ear and PFSR disease score recorded nonsignificant positive correlation with cob weight with husk at both levels. Days to 50\% tasseling recorded non-significant negative at phenotypic and significant negative correlation at genotypic level with cob weight with husk. Days to $50 \%$ silking and total soluble sugars recorded non-significant negative correlations and green fodder yield recorded significant positive at phenotypic and significant negative correlation at genotypic level with cob weight with husk. The results were in agreement with the findings of Raghu et al., (2011), Reddy et al., (2013), Suhasini et al., (2016) and Niji et al., (2018) for the traits plant height, ear height, ear length, ear girth and no. of kernel rows per ear, Raghu et al., (2011) and Suhasini et al., (2016) for the trait no. of kernels per row, Kumara et al., (2011) and Chinthiya et al., (2019) for traits plant height, cob length, cob diameter and no. of kernels per row. Hence simultaneous selection can be done in these positively correlated traits through further breeding programmes.

Inter correlations among yield components revealed that days to $50 \%$ silking recorded significant positive correlation with days to $50 \%$ tasseling. Similarly, ear height with plant height, trait ear length with plant height, trait ear diameter with plant height, ear height and ear length, trait number of kernel rows per ear 
with ear height and ear diameter, trait number of kernels per row with ear length and ear diameter, trait cob weight without husk with plant height, ear height, ear length, ear diameter and no. of kernels per row recorded significant positive correlations.

Table.1 Analysis of variance for yield and yield attributing traits in 45 sweet corn inbred lines

\begin{tabular}{|l|c|c|c|}
\hline \multirow{2}{*}{\multicolumn{1}{|c|}{ Source of variation }} & \multicolumn{3}{c|}{ Mean sum of squares of } \\
\cline { 2 - 4 } & Replications & Genotypes & Error \\
\hline Days to 50\% tasseling & 1 & 24 & 24 \\
\hline Days to 50\% silking & 0.02 & 13.6 & 8.1 \\
\hline Plant height (cm) & 2 & $14.4^{* *}$ & 2.54 \\
\hline Ear height (cm) & 1.3 & 565.8 & 293.8 \\
\hline Ear length (cm) & 0.7 & $177.8^{* *}$ & 0.7 \\
\hline Ear diameter (cm) & $2.2^{*}$ & $10.3^{* *}$ & 0.3 \\
\hline No. of kernel rows per ear & 2 & $3.0^{* *}$ & 0.5 \\
\hline No. of kernels per row & 0 & $5.9^{* *}$ & 0.6 \\
\hline Cob weight with husk (kg/ha) & 5.1 & $44.0^{* *}$ & 1.4 \\
\hline Cob weight without husk (kg/ha) & 492523.3 & $27460145.0^{* *}$ & 972427.9 \\
\hline Total soluble sugars (\%) & 3843 & $24726071.6^{* *}$ & 6214186.9 \\
\hline Green fodder yield (kg/ha) & 0.7 & $3.5^{* *}$ & 0.2 \\
\hline PFSR disease score & 1201647 & 12327470 & 23217966 \\
\hline
\end{tabular}

$*$, ** Significant at 5\% \& 1\% level respectively.

Table.2 Genetic parameters for yield and yield attributing traits in sweet corn

\begin{tabular}{|c|c|c|c|c|c|c|c|c|}
\hline \multirow[t]{2}{*}{ Character } & \multirow[t]{2}{*}{ Mean } & \multicolumn{2}{|c|}{ Range } & \multirow{2}{*}{$\begin{array}{r}\text { PCV } \\
(\%)\end{array}$} & \multirow{2}{*}{$\begin{array}{c}\text { GCV } \\
(\%)\end{array}$} & \multirow{2}{*}{$\begin{array}{c}\text { Heritability } \\
(\%) \\
\text { in broad } \\
\text { sense }\end{array}$} & \multirow[t]{2}{*}{ GA } & \multirow{2}{*}{$\begin{array}{c}\text { GAM } \\
(\%)\end{array}$} \\
\hline & & Minimum & Maximum & & & & & \\
\hline Days to $50 \%$ tasseling & 74 & 70 & 79 & 4.43 & 2.23 & 25 & 1.72 & 2.32 \\
\hline Days to $50 \%$ silking & 77 & 73 & 82 & 3.79 & 3.18 & 70 & 4.21 & 5.48 \\
\hline Plant height (cm) & 143 & 119 & 175 & 14.49 & 8.15 & 31 & 13.51 & 9.44 \\
\hline Ear height (cm) & 47 & 32 & 67 & 20.29 & 20.21 & 99 & 19.3 & 41.45 \\
\hline Ear length (cm) & 15 & 10 & 20 & 15.1 & 14.56 & 93 & 4.43 & 28.94 \\
\hline Ear diameter (cm) & 12 & 10 & 15 & 10.96 & 9.07 & 68 & 1.9 & 15.47 \\
\hline $\begin{array}{l}\text { No. of kernel rows per } \\
\text { ear }\end{array}$ & 14 & 12 & 20 & 12.32 & 11.01 & 79 & 2.98 & 20.25 \\
\hline No. of kernels per row & 27 & 18 & 37 & 17.74 & 17.15 & 93 & 9.18 & 34.15 \\
\hline $\begin{array}{l}\text { Cob weight with husk } \\
\text { (kg/ha) }\end{array}$ & 17329 & 10429 & 25408 & 21.75 & 21 & 93 & 7235.83 & 41.75 \\
\hline $\begin{array}{l}\text { Cob weight without husk } \\
\text { (kg/ha) }\end{array}$ & 13267 & 6581 & 20211 & 29.64 & 22.93 & 59 & 4847.75 & 36.53 \\
\hline Total soluble sugars (\%) & 14.1 & 11.7 & 17.2 & 9.76 & 9.14 & 87 & 2.49 & 17.62 \\
\hline $\begin{array}{l}\text { Green fodder yield } \\
\text { (kg/ha) }\end{array}$ & 30771 & 24220 & 35072 & 13.7 & 7.58 & -30 & -2660.77 & -8.64 \\
\hline PFSR disease score & 4.8 & 3 & 7.5 & 30.34 & 27.94 & 84 & 2.58 & 53.01 \\
\hline
\end{tabular}


Table.3 Phenotypic (P) and genotypic $(\mathrm{G})$ correlations for yield and yield attributing traits in sweet corn

\begin{tabular}{|c|c|c|c|c|c|c|c|c|c|c|c|c|c|c|}
\hline Character & & $\begin{array}{c}\text { Days to } \\
50 \% \\
\text { tasseling }\end{array}$ & $\begin{array}{c}\text { Days to } \\
50 \% \\
\text { silking }\end{array}$ & $\begin{array}{c}\text { Plant } \\
\text { height } \\
(\mathbf{c m})\end{array}$ & $\begin{array}{c}\text { Ear } \\
\text { height } \\
(\mathrm{cm})\end{array}$ & $\begin{array}{l}\text { Ear } \\
\text { length } \\
\text { (cm) }\end{array}$ & $\begin{array}{c}\text { Ear } \\
\text { diameter } \\
(\mathbf{c m})\end{array}$ & $\begin{array}{l}\text { Number } \\
\text { of rows } \\
\text { per ear }\end{array}$ & $\begin{array}{c}\text { Number } \\
\text { of kernels } \\
\text { per row }\end{array}$ & $\begin{array}{c}\text { Cob } \\
\text { weight } \\
\text { without } \\
\text { husk } \\
\text { (kg/ha) }\end{array}$ & $\begin{array}{c}\text { Total } \\
\text { soluble } \\
\text { sugars } \\
(\%)\end{array}$ & $\begin{array}{c}\text { Green } \\
\text { fodder } \\
\text { yield } \\
\text { (kg/ha) }\end{array}$ & $\begin{array}{c}\text { PFSR } \\
\text { disease } \\
\text { score }\end{array}$ & $\begin{array}{c}\text { Cob } \\
\text { weight } \\
\text { with husk } \\
\text { (kg/ha) }\end{array}$ \\
\hline \multirow{2}{*}{$\begin{array}{l}\text { Days to } 50 \% \\
\text { tasseling }\end{array}$} & $\mathbf{P}$ & 1 & $0.9484 * *$ & 0.1542 & -0.1192 & -0.2099 & -0.0839 & 0.1067 & -0.266 & 0.163 & 0.2458 & $0.5327 * *$ & 0.0579 & -0.0234 \\
\hline & G & 1 & $1.1808 * *$ & $-1.9185 * *$ & -0.1833 & $-0.5130 * *$ & $-1.1882 * *$ & 0.0513 & $-0.5684 * *$ & $-0.9559 * *$ & $0.5463 * *$ & $1.5505^{* *}$ & $0.3268^{*}$ & $-0.4538 * *$ \\
\hline \multirow{2}{*}{$\begin{array}{l}\text { Days to } 50 \% \\
\text { silking }\end{array}$} & $\mathbf{P}$ & & 1 & -0.0254 & -0.1395 & -0.2349 & -0.2222 & 0.0935 & $-0.2831^{*}$ & 0.0498 & 0.2746 & $0.3478^{*}$ & 0.0677 & -0.0649 \\
\hline & $\mathbf{G}$ & & 1 & $-0.9187 * *$ & -0.148 & $-0.3191^{*}$ & $-0.6691 * *$ & 0.0303 & $-0.3508 *$ & $-0.4042 * *$ & $0.3550 *$ & $0.4637 * *$ & 0.1753 & -0.2259 \\
\hline \multirow[t]{2}{*}{ Plant height $(\mathbf{c m})$} & $\mathbf{P}$ & & & 1 & $0.6733 * *$ & $0.3693 * *$ & $0.6601 * *$ & 0.1437 & 0.2677 & $0.6576^{* *}$ & -0.0142 & $0.6113 * *$ & 0.0212 & $0.5503 * *$ \\
\hline & G & & & 1 & $1.2497 * *$ & $0.6258^{* *}$ & $0.5740 * *$ & 0.2002 & $0.4749 * *$ & $0.3229^{*}$ & 0.0002 & $1.0573 * *$ & 0.2232 & $0.6752 * *$ \\
\hline \multirow[t]{2}{*}{ Ear height (cm) } & $\mathbf{P}$ & & & & 1 & 0.2785 & $0.5410^{* *}$ & $0.3383^{*}$ & 0.1306 & $0.3478^{*}$ & 0.1979 & 0.1812 & 0.2193 & $0.4062^{* *}$ \\
\hline & G & & & & 1 & $0.2870^{*}$ & $0.6674 * *$ & $0.3936^{* *}$ & 0.1258 & $0.4776 * *$ & 0.1977 & $-0.3959 * *$ & 0.2311 & $0.4260 * *$ \\
\hline \multirow[t]{2}{*}{ Ear length (cm) } & $\mathbf{P}$ & & & & & 1 & $0.3847 * *$ & -0.2119 & $0.8368 * *$ & $0.6613 * *$ & -0.06 & $0.3270^{*}$ & $0.2914 *$ & $0.7153 * *$ \\
\hline & G & & & & & 1 & $0.4122 * *$ & -0.2663 & $0.8590 * *$ & $0.8369 * *$ & -0.0802 & $-0.5394 * *$ & $0.3553^{*}$ & $0.7287 * *$ \\
\hline \multirow[t]{2}{*}{ Ear diameter (cm) } & $\mathbf{P}$ & & & & & & 1 & $0.4637 * *$ & $0.4084 * *$ & $0.6601 * *$ & -0.0281 & $0.5241 * *$ & $0.2971^{*}$ & $0.6395^{* *}$ \\
\hline & G & & & & & & 1 & $0.5450 * *$ & $0.4573^{* *}$ & $0.5457 * *$ & -0.0311 & 0.0454 & $0.4874 * *$ & $0.6387 * *$ \\
\hline \multirow{2}{*}{$\begin{array}{c}\text { Number of rows } \\
\text { per ear }\end{array}$} & $\mathbf{P}$ & & & & & & & 1 & -0.1966 & 0.0298 & 0.0204 & 0.0367 & 0.2505 & 0.0393 \\
\hline & $\mathbf{G}$ & & & & & & & 1 & -0.2389 & -0.0458 & 0.0383 & 0.0551 & $0.3046^{*}$ & 0.0111 \\
\hline \multirow{2}{*}{$\begin{array}{c}\text { Number of kernels } \\
\text { per row }\end{array}$} & $\mathbf{P}$ & & & & & & & & 1 & $0.6495 * *$ & -0.2412 & $0.2964 *$ & 0.107 & $0.6999 * *$ \\
\hline & $\mathbf{G}$ & & & & & & & & 1 & $0.8511^{* *}$ & $-0.2958^{*}$ & $-0.5438 * *$ & 0.1401 & $0.7290 * *$ \\
\hline \multirow{2}{*}{$\begin{array}{c}\text { Cob weight } \\
\text { without husk } \\
(\mathrm{kg} / \mathrm{ha})\end{array}$} & $\mathbf{P}$ & & & & & & & & & 1 & -0.155 & $0.6748 * *$ & 0.1636 & $0.9487 * *$ \\
\hline & G & & & & & & & & & 1 & -0.2003 & 0.0924 & $0.3309^{*}$ & $1.0719^{* *}$ \\
\hline \multirow{2}{*}{$\begin{array}{c}\text { Total soluble } \\
\text { sugars }(\%)\end{array}$} & $\mathbf{P}$ & & & & & & & & & & 1 & -0.0365 & $0.3826 * *$ & -0.175 \\
\hline & G & & & & & & & & & & 1 & 0.0129 & $0.3578^{*}$ & -0.1945 \\
\hline \multirow{2}{*}{$\begin{array}{l}\text { Green fodder yield } \\
\text { (kg/ha) }\end{array}$} & $\mathbf{P}$ & & & & & & & & & & & 1 & 0.0364 & $0.4760 * *$ \\
\hline & $\mathbf{G}$ & & & & & & & & & & & 1 & $-0.3332^{*}$ & $-0.4152 * *$ \\
\hline \multirow[t]{2}{*}{ PFSR disease score } & $\mathbf{P}$ & & & & & & & & & & & & 1 & 0.1882 \\
\hline & G & & & & & & & & & & & & 1 & 0.2569 \\
\hline
\end{tabular}


Table.4 Phenotypic (P) and genotypic $(\mathrm{G})$ path coefficients for yield and yield attributing traits in sweet corn

\begin{tabular}{|c|c|c|c|c|c|c|c|c|c|c|c|c|c|c|}
\hline Character & & $\begin{array}{c}\text { Days to } \\
50 \% \\
\text { tasseling }\end{array}$ & $\begin{array}{c}\text { Days to } \\
50 \% \\
\text { silking }\end{array}$ & $\begin{array}{c}\text { Plant } \\
\text { height } \\
\text { (cm) }\end{array}$ & $\begin{array}{c}\text { Ear } \\
\text { height } \\
\text { (cm) }\end{array}$ & $\begin{array}{c}\text { Ear } \\
\text { length } \\
\text { (cm) }\end{array}$ & $\begin{array}{c}\text { Ear } \\
\text { diameter } \\
(\mathbf{c m})\end{array}$ & $\begin{array}{c}\text { Number } \\
\text { of rows } \\
\text { per ear }\end{array}$ & $\begin{array}{c}\begin{array}{c}\text { Number } \\
\text { of } \\
\text { kernels }\end{array} \\
\text { per row }\end{array}$ & $\begin{array}{c}\text { Cob } \\
\text { weight } \\
\text { without } \\
\text { husk } \\
\text { (kg/ha) }\end{array}$ & $\begin{array}{c}\text { Total } \\
\text { soluble } \\
\text { sugars } \\
(\%)\end{array}$ & $\begin{array}{c}\text { Green } \\
\text { fodder } \\
\text { yield } \\
\text { (kg/ha) }\end{array}$ & $\begin{array}{c}\text { PFSR } \\
\text { disease } \\
\text { score }\end{array}$ & $\begin{array}{c}\text { Cob } \\
\text { weight } \\
\text { with husk } \\
\text { (kg/ha) }\end{array}$ \\
\hline \multirow{2}{*}{$\begin{array}{c}\text { Days to } 50 \% \\
\text { tasseling }\end{array}$} & $\mathbf{P}$ & -0.4783 & -0.4536 & -0.0738 & 0.0570 & 0.1004 & 0.0401 & -0.0510 & 0.1272 & -0.0779 & -0.1175 & -0.2548 & -0.0277 & -0.0234 \\
\hline & G & -0.7218 & -0.8524 & 1.3848 & 0.1323 & 0.3703 & 0.8577 & -0.0371 & 0.4103 & 0.6900 & -0.3944 & -1.1192 & -0.2359 & $-0.4538 * *$ \\
\hline \multirow{2}{*}{$\begin{array}{c}\text { Days to } 50 \% \\
\text { silking }\end{array}$} & $\mathbf{P}$ & 0.4355 & 0.4592 & -0.0117 & -0.0640 & -0.1079 & -0.1020 & 0.0429 & -0.1300 & 0.0229 & 0.1261 & 0.1597 & 0.0311 & -0.0649 \\
\hline & $\mathbf{G}$ & 1.1549 & 0.9780 & -0.8985 & -0.1447 & -0.3121 & -0.6544 & 0.0296 & -0.3431 & -0.3954 & 0.3472 & 0.4535 & 0.1714 & -0.2259 \\
\hline \multirow[t]{2}{*}{ Plant height (cm) } & $\mathbf{P}$ & -0.0193 & 0.0032 & -0.1252 & -0.0843 & -0.0462 & -0.0826 & -0.0180 & -0.0335 & -0.0823 & 0.0018 & -0.0765 & -0.0027 & $0.5503 * *$ \\
\hline & G & -0.1649 & -0.0790 & 0.0860 & 0.1074 & 0.0538 & 0.0493 & 0.0172 & 0.0408 & 0.0278 & 0.0000 & 0.0909 & 0.0192 & $0.6752 * *$ \\
\hline \multirow[t]{2}{*}{ Ear height (cm) } & $\mathbf{P}$ & -0.0112 & -0.0131 & 0.0632 & 0.0938 & 0.0261 & 0.0508 & 0.0317 & 0.0123 & 0.0326 & 0.0186 & 0.0170 & 0.0206 & $0.4062 * *$ \\
\hline & $\mathbf{G}$ & 0.0174 & 0.0140 & -0.1183 & -0.0947 & -0.0272 & -0.0632 & -0.0373 & -0.0119 & -0.0452 & -0.0187 & 0.0375 & -0.0219 & $0.4260 * *$ \\
\hline \multirow[t]{2}{*}{ Ear length (cm) } & $\mathbf{P}$ & -0.0232 & -0.0260 & 0.0409 & 0.0308 & 0.1107 & 0.0426 & -0.0235 & 0.0927 & 0.0732 & -0.0066 & 0.0362 & 0.0323 & $0.7153 * *$ \\
\hline & G & 0.0027 & 0.0017 & -0.0033 & -0.0015 & -0.0053 & -0.0022 & 0.0014 & -0.0046 & -0.0045 & 0.0004 & 0.0029 & -0.0019 & $0.7287 * *$ \\
\hline \multirow[t]{2}{*}{ Ear diameter (cm) } & $\mathbf{P}$ & -0.0122 & -0.0324 & 0.0961 & 0.0788 & 0.0560 & 0.1456 & 0.0675 & 0.0595 & 0.0961 & -0.0041 & 0.0763 & 0.0433 & $0.6395 * *$ \\
\hline & $\mathbf{G}$ & 0.0667 & 0.0376 & -0.0322 & -0.0375 & -0.0231 & -0.0561 & -0.0306 & -0.0257 & -0.0306 & 0.0017 & -0.0025 & -0.0274 & $0.6387 * *$ \\
\hline \multirow{2}{*}{$\begin{array}{l}\text { Number of rows } \\
\text { per ear }\end{array}$} & $\mathbf{P}$ & & & & & & & & & & -0.0008 & -0.0014 & -0.0093 & 0.0393 \\
\hline & G & 0.0037 & 0.0022 & 0.0146 & 0.0287 & -0.0194 & 0.0397 & 0.0729 & -0.0174 & -0.0033 & 0.0028 & 0.0040 & 0.0222 & 0.0111 \\
\hline \multirow{2}{*}{$\begin{array}{l}\text { Number of kernels } \\
\text { per row }\end{array}$} & $\mathbf{P}$ & 0.0178 & 0.0189 & -0.0179 & -0.0087 & -0.0559 & -0.0273 & 0.0131 & -0.0668 & -0.0434 & 0.0161 & -0.0198 & -0.0072 & $0.6999 * *$ \\
\hline & G & 0.0597 & 0.0368 & -0.0498 & -0.0132 & -0.0902 & -0.0480 & 0.0251 & -0.1050 & -0.0893 & 0.0310 & 0.0571 & -0.0147 & $0.7290 * *$ \\
\hline \multirow{2}{*}{$\begin{array}{c}\text { Cob weight } \\
\text { without husk } \\
\text { (kg/ha) }\end{array}$} & $\mathbf{P}$ & 0.1693 & 0.0517 & 0.6832 & 0.3613 & 0.6870 & 0.6858 & 0.0310 & 0.6747 & 1.0389 & -0.1611 & 0.7011 & 0.1700 & $0.9487 * *$ \\
\hline & $\mathbf{G}$ & -0.8710 & -0.3683 & 0.2942 & 0.4352 & 0.7626 & 0.4972 & -0.0418 & 0.7755 & 0.9112 & -0.1825 & 0.0842 & 0.3015 & $1.0719 * *$ \\
\hline \multirow{2}{*}{$\begin{array}{c}\text { Total soluble } \\
\text { sugars }(\%)\end{array}$} & $\mathbf{P}$ & -0.0092 & -0.0102 & 0.0005 & -0.0074 & 0.0022 & 0.0010 & -0.0008 & 0.0090 & 0.0058 & -0.0372 & 0.0014 & -0.0142 & -0.175 \\
\hline & G & 0.0021 & 0.0014 & 0.0000 & 0.0008 & -0.0003 & -0.0001 & 0.0001 & -0.0012 & -0.0008 & 0.0039 & 0.0001 & 0.0014 & -0.1945 \\
\hline \multirow{2}{*}{$\begin{array}{c}\text { Green fodder yield } \\
(\mathrm{kg} / \mathrm{ha})\end{array}$} & $\mathbf{P}$ & -0.0861 & -0.0562 & -0.0989 & -0.0293 & -0.0529 & -0.0848 & -0.0059 & -0.0479 & -0.1091 & 0.0059 & -0.1617 & -0.0059 & $0.4760 * *$ \\
\hline & G & -0.0161 & -0.0048 & -0.0110 & 0.0041 & 0.0056 & -0.0005 & -0.0006 & 0.0056 & -0.0010 & -0.0001 & -0.0104 & 0.0035 & $-0.4152 * *$ \\
\hline \multirow{2}{*}{$\begin{array}{l}\text { PFSR disease } \\
\text { score }\end{array}$} & $\mathbf{P}$ & -0.0024 & -0.0028 & -0.0009 & -0.0092 & -0.0122 & -0.0125 & -0.0105 & -0.0045 & -0.0069 & -0.0161 & -0.0015 & -0.0420 & 0.1882 \\
\hline & G & 0.0129 & 0.0069 & 0.0088 & 0.0091 & 0.0140 & 0.0192 & 0.0120 & 0.0055 & 0.0130 & 0.0141 & -0.0131 & 0.0394 & 0.2569 \\
\hline
\end{tabular}

Phenotypic and genotypic residual effects 0.1340 and 0.0264 respectively. 
Green fodder yield recorded significant positive correlation with days to $50 \%$ tasseling, days to $50 \%$ silking and plant height; PFSR disease score recorded significant positive correlation with total soluble sugars, ear length and ear diameter. Whereas, total soluble sugars recorded significant positive genotypic correlation with days to $50 \%$ tasseling and days to $50 \%$ silking. These traits can be improved through simultaneous selection of other traits.

High correlation coefficients may not be always giving the true picture or could mislead the decision because the correlation between two variables may be due to a third factor. Therefore, it is necessary to analyze the cause and effect relationship between dependent and independent variables to reveal the nature of relationship between the variables.

Path coefficient analysis revealed that the trait days to $50 \%$ silking recorded highest direct effect (0.9780) followed by cob weight without husk (0.9112), plant height (0.0860), no. of kernel rows per ear (0.0729), PFSR disease score (0.0394) and total soluble sugars (0.0039) (Table 4) at genotypic level and these characters are significantly associated with cob weight with husk. Similar results were obtained in findings of Raghu et al., (2011), Reddy et al., (2013) and Begum et al., (2016) for plant height and no. of kernel rows per ear, Ilker (2011) for ear weight and no. of kernels rows per ear, Sadaiah et al., (2014) for sugar content and Pavlov et al., (2015) for plant height. Thus direct selection for these traits would be effective i.e., with slight increase in one of the above traits may directly contribute to increase in cob weight with husk.

On the other hand, days to $50 \%$ tasseling, ear height, ear length, ear diameter, no. of kernels per row and green fodder yield exhibited negative direct effect on cob weight with husk. These findings were in harmony with the findings of Raghu et al., (2011) for days to $50 \%$ tasseling, ear length and ear height, Begum et al., (2016) for days to 50\% tasseling and ear height Dan Singh Jakhar et al., (2017) for ear height. Most of the traits exhibited indirect influence on cob weight with husk through days to $50 \%$ tasseling, days to $50 \%$ silking, plant height, ear height, ear length, ear diameter, no. of kernel rows per ear, no. of kernels per row and total soluble sugars. The results thus emphasize that selection could be more effective by indirect selection of these traits.

Phenotypic and genotypic residual effects were 0.1340 and 0.0264 respectively, indicating that some characters which had due weightage in selection for yield improvement are to be included.

From the findings of the present investigation, it may be concluded that characters ear height, ear length, number of kernel rows per ear, number of kernels per row, cob weight without husk and PFSR disease score were important for selection of sweet corn genotypes as these traits recorded moderate to high range of GCV, PCV, heritability, genetic advance over mean and exhibited positive significant correlations and some of these traits also had direct effects on cob weight with husk i.e. green cob yield.

\section{References}

Alan, O., Kinaci, G., Kinaci, E., Kutlu, I., Budak Basciftci, Z., Sonmez, K., Evrenosoglu. Y. 2013. Genetic variability and association analysis of some quantitative characters in sweet corn. Notulae Botanicae Horti Agrobotanici. 41(2):404-413.

Ayodeji, Abe and Comfort, Adeola. 2019. Genetic variability, heritability and 
genetic advance in shrunken-2 supersweet corn (Zea mays L. saccharata) populations. Journal of Plant Breeding and Crop Science. 11. 100-105. 10.5897/JPBCS2018.0799.

Begum, S., Ahmed, A., Omy, S. H., Rohman, M. M., and Amiruzzaman, M. 2016. Genetic variability, character association and path analysis in maize (Zea mays L.). Bangladesh Journal of Agricultural Research, 41(1), 173-182

Bello, O.B., Ige, S.A., Azeez, M.A., Afolabi, M.S., Abdulmaliq, S.Y., Mahamood, J. 2012. Heritability and genetic advance for grain yield and its component characters in maize (Zea mays L.). International Journal of Plant Research. 2(5):138- 145.

Bilgin, O., Korkut, K.Z., Baser, I., Dalioglu, O., Ozturk, I., Kahraman, T., Balkan, A. 2010. Variation and Heritability for Some Semolina Characteristics and Grain Yield and their Relations in Durum Wheat (Triticum durum Desf.).World J Agric Sci. 6: 301- 308

Burton, G. M. 1952. Quantitative inheritance in grasses. Proceedings of 6 th International Grassland Cong., 1: 277283.

Chinthiya, A., Ganesan, K., Ravikesavan, R and Senthil, N. 2019. Combining ability and association studies on different yield contributing traits for enhanced green cob yield in sweet corn (Zea mays con Var saccharata). Electronic Journal of Plant Breeding. 10(2): 500-511.

Dan Singh Jakhar, Rajesh Singh and Amit Kumar. 2017. Studies on Path Coefficient Analysis in Maize (Zea mays L.) for Grain Yield and Its Attributes.

Int.J.Curr.Microbiol.App.Sci. 6(4): 2851-2856.

Dewanto, V., Wu, X and Liu, R.H. 2002. Processed sweet corn has higher antioxidant activity. Journal of
Agriculture and Food Chemistry. 50(14): 4959-4964.

Dewey, D.R. and Lu, K.H. 1959. A correlation and path coefficient analysis of components of crested wheat grass seed production. Agron. J., 51: 515-518.

Hanson, C. H., Robinson, H. F. and Comstock, R. E. 1956. Biometrical studies of yield in segregating populations of Korean Lespedeza. Agron. J. 48: 268-272.

Hefny, M. 2011. Genetic parameters and path analysis of yield and its components in corn inbred lines (Zea mays L.) at different sowing dates. Asian Journal of Crop Science. 3(3):106-117.

Ibrahim, K.E and Juvik, J.A. 2009. Feasibility for improving phytonutrient content in vegetable crops using conventional breeding strategies: Case study with carotenoids and tocopherols in sweet corn and broccoli. Journal of Agricultural and Food Chemistry. 57(11), 4636-4644.

Ilker, E. 2011. Correlation and path coefficient analyses in sweet corn. Turkish Journal of field crops, 16(2), 105-107.

Johnson, H.W., Robinson, H.F and Comstock, R.E. 1955. Estimation of genetic and environmental variability in soybeans. Agronomy Journal. 47: 314-318.

Kumara, S. 2011.Genetic studies on hybrids and their inbreds of sweet corn (Zea mays convar saccharata). (M.Sc.), Tamil Nadu Agricultural University, Coimbatore, India.

Sadaiah. K. and Reddy, V and Kumar, S and Ganesh, M. 2014. Path analysis studies in sweet corn (Zea mays L. saccharata). Green farming. 5(1): 98-100.

Meena, M., Singh, R., and Meena, H. 2016. Genetic variability, heritability and genetic advance studies in newly developed maize genotypes (Zea mays L.). The Bioscan. 11(3): 1787-1791. 
Niji, M.S., Ravikesavan, R., Ganesan, K.N and Chitdeshwari, T. 2018. Genetic variability, heritability and character association studied in sweet corn (Zea mays L. saccharata). Electronic Journal of Plant Breeding. 9(3):1038-1044.

Nzuve, F., Githiri, S., Mukunya, D.M and Gethi, J. (2014). Genetic variability and correlation studies of grain yield and related agronomic traits in maize. Journal of Agricultural Science. 6(9):166-176.

Panse, V.G., Sukhatme P.V. 1985 Statistical methods for agricultural workers. Indian Council of Agricultural research, New Delhi.

Pavlov, J., Delić, N., Marković, K., Crevar, M., Čamdžija, Z., and Stevanović, M. 2015. Path analysis for morphological traits in maize (Zea mays L.). Genetika, 47(1), 295-301.

Raghu, B., Suresh, J., Sudheer Kumar, S. and Saidaiah, P. 2011. Character association and path analysis in maize (Zea mays L.). Madras Agricultural Journal. 98 (1-3): 7-9.

Reddy, V. R., Jabeen, F., Sudarshan, M. R., and Rao, A. S. 2012. Studies on genetic variability, heritability, correlation and path analysis in maize (Zea mays L.) over locations. International Journal of Applied Biology and Pharmaceutical Technology, 4(1), 196-199.

Sadaiah, K., Reddy, V. N., and Kumar, S. S.
2013. Correlation studies for yield and yield contributing characters in Sweetcorn (Zea mays L. saccharata). Int J Agric Inno Res, 2(2), 145-148.

Sankar, P.D., Sheeba, A and Anbumalarmathi, J., 2006. Variability and Character Association Studies in Rice. Agric Sci Digest. 26: 182-184

Sesay, S., Ojo, D., Ariyo, O.J and Meseka, S. 2016. Genetic variability, heritability and genetic advance studies in top-cross and three-way cross maize (Zea mays L.) hybrids. Maydica. 61(2).

Singh, R.K., Chaudhary, B.D., 1985. Biometrical Methods in Quantitative Genetic Analysis, pp. 318. Kalayani Publishers, New Delhi, India

Sivasubramanian, V. and Madhavamenon, P. 1973. Path analysis for yield and yield components of rice. Madras Ag. Journal. 60: 217-1221.

Suhaisini, B., Ravikesavan, R., and Yuvaraja, A. 2016. Genetic Variability and Correlation among Yield and Yield Contributing Traits in Sweet Corn. Madras Agricultural Journal. 103.

Tracy, W.F. 1994. Sweet corn. In: Hallauer (ed.). Speciality types of maize. CRC Press, Boca Raton, Florida. p. 147-187.

Wright, S. 1921. Correlation and causation. $J$. Agric. Res. 20: 557-585

Wright, S. 1929. Path coefficients and path regression: Alternative complementary concepts. Biometric. 16: 189-202.

\section{How to cite this article:}

Sonal Chavan, D. Bhadru, V. Swarnalatha and Mallaiah, B. 2020. Studies on Genetic Parameters, Correlation and Path Analysis for Yield and Yield Attributing Traits in Sweet Corn (Zea mays L. saccharata). Int.J.Curr.Microbiol.App.Sci. 9(07): 1725-1734. doi: https://doi.org/10.20546/ijcmas.2020.907.199 